\title{
Marca Cabo Verde. Aproximación al reciente posicionamiento del país a través de su identidad turística
}

Edson Redy Moreira dos Santos | edson.santos@docente.unicv.edu.cv (Escola de Negocios e Governacao -Universidade de Cabo Verde)

Licenciado en Lengua, Literatura y CULtURA - Especialidad EN Estudios Ingleses (UNiversidad de Cabo Verde). Master en Gestión Turística en el instituto Superior de Ciencias Empresariales y Turismo (Oporto-Portugal, 2013). profesor del Departamento de Ciencias Sociais e humanas (UNIVERSIDADE DE CABO VeRDE).

Lorena Rodríguez Campo | lorenarcampo@uvigo.es

(UNIVERSIDAD DE VIGO)

Doble Licenciatura en ADE (UDC, 1998) e ITM (UOC, 2011). Doctora en Economía (2010) con accésit a la Mejor tesis doctoral (Aemark) y Mejor Paper (Aecit). Profesora de Comercialización e investigación de Mercados (Uvigo) y de diversos postgrados Vigo, A Coruña, Oporto y MÉxico. Anteriormente, Gerente de la Productora Filmanova (2000-2005).

Resumen: Desde que comenzó el siglo XXI, Cabo Verde presenta crecimientos anuales de turistas del $17,5 \%$ lo que le ha consolidado como país emergente y estratégico. Debido a ello, el Ministerio de Turismo en 2010, ha tratado de desarrollar estrategias de marketing para el destino. Una revisión crítica y reflexiva a través del análisis DAFO, tanto de la creación de la identidad visual como de las actuaciones derivadas del Plan Estratégico para el Desarrollo del Turismo, permiten concluir que las autoridades han escogido un posicionamiento erróneo carente de autenticidad siendo necesario y posible el reposicionar Cabo Verde pues posee características fundamentales para constituirse en una marca diferenciada $y$ única. experiencial

Palabras clave: marca Cabo Verde, identidad turística, Branding de destinos, marca

Abstract: Since the beginning of the 21st century, Cape Verde has had an annual growth of $17.5 \%$ of tourist arrivals, which in turn, has established the country as a strategic emerging destination. As a result, of this, in 2010 the Ministry of Tourism attempted to develop a marketing strategy for the destination. A critical and reflective analysis anchored by a SWOT Analysis based on both, the creation of the visual identity as well as the actions undertaken in the National Strategic Plan for Tourism Development concluded that the visual identity selected by authorities lack the fundamental authentic characteristics necessary to position Brand Cape Verde as a differentiated and unique visual identity.

Key words: Brand Cape Verde, tourism identity, destination branding, experiential brand 


\section{Introducción}

La industria del turismo en Cabo Verde ha sido reconocida por el gobierno como un sector estratégico clave en el desarrollo socioeconómico del país. En 2012, el turismo a nivel mundial tuvo un crecimiento del 3,8\% con respecto a 2011 (OMT, 2013). Las estadísticas regionales muestran que, en el mismo período, la región del África subsahariana recibió 33,8 millones de llegadas de turistas (un 5\% más). Mientras Cabo Verde, obtuvo un extraordinario crecimiento del 30,2\% de visitantes en relación al 2011 procedentes, principalmente, de Europa (Reino Unido, Alemania, Bélgica, Holanda, Portugal y Francia). El atractivo turístico del país radica en su producto sol y playa. Además, cuenta con cuatro aeropuertos internacionales y unos rasgos distintivos en cada una de las diez islas que componen el archipiélago, que permiten su promoción en el ámbito del turismo cultural (Mitchell, 2008: 3).

En 1975, el país obtiene la independencia de los portugueses y se proclama la República de Cabo Verde. Situada en el norte del Océano Atlántico, a unos 500 kilómetros de la costa de África Occidental y vecina de Senegal, Gambia y Mauritania, su ubicación geoestratégica que conecta Europa, África y América sirve de puente entre culturas y continentes. Igualmente, este país ofrece para constituirse en un destino emergente y atractivo, un clima relajante con acceso a enormes playas de arena y aguas cálidas, la calidad del alojamiento, un entorno ideal, la diversidad cultural, gente hospitalaria y la posibilidad de realizar actividades de interés especial, tales como: buceo, escalada, senderismo, pesca deportiva y barranquismo (DGT, 2010a). Por todo ello, en 2010 el gobierno pone en marcha el Plan Estratégico para el Desarrollo del Turismo (PEDT) y la creación de una identidad turística de Cabo Verde conducente a identificar los productos del país, fortalecer su competitividad y garantizar su permanencia en el mercado internacional.

Como indica Tkachuk (2008), el citymarketing se divide en dos ejes: el endomarketing, donde el objetivo apunta a identificar las principales necesidades de los residentes a fin de que puedan verse autorrepresentados $y$, por lo tanto, la relación ciudadano-territorio se plasma a partir de la identidad; $y$, el exomarketing donde el objetivo se centra en generar las condiciones adecuadas para que turistas e inversores se sientan convocados por el atractivo que la ciudad les ofrece y donde la relación turista-territorio se da por medio fundamentalmente de la imagen. Por su parte Hall (2010), afirma que las marcas contribuyen claramente a la construcción de la ventaja competitiva para el territorio siendo importante, para ello, hacer hincapié en las estrategias de marketing interno (o endomarketing) a la hora de desarrollar la marca y comunicar la identidad de un lugar incluyendo el orgullo de la comunidad local y elementos del entorno físico. Según Muñiz y Cervantes (2010), posteriormente se produce la creación de la comunicación urbana a través de elementos como lemas y logotipos y se desarrollan las estrategias de comercialización del destino, principalmente, hacia mercados externos (exomarketing). 
El objetivo principal de este trabajo es analizar el proceso de branding y elaborar una matriz DAFO de la marca Cabo Verde. Para ello se comienza por la revisión teórica del concepto de marca y de branding de destinos y su aplicación al caso objeto de estudio. Posteriormente, se efectúa un análisis de la identidad de la marca Cabo Verde y se elabora un DAFO como herramienta estratégica para conocer la situación real en la que se encuentra el destino y definir el posicionamiento estratégico.

\section{Concepto de marca y de branding de destinos}

La marca se puede definir como un nombre distintivo y/o símbolo destinado a identificar los bienes y servicios de cualquier vendedor o grupo de vendedores, y para diferenciar los productos de los de sus competidores (Aaker, 1991; Keller, 2003; Kotler y Gertner, 2004; Kotler, Bowen y Makens, 2010). Además, es un conjunto de valores funcionales y emocionales que prometen una experiencia única y agradable entre un comprador y un vendedor (Lynch y De Chernatony, 2004; Dinnie, 2008: 14).

De Chernatony y Dall'Olmo (1998) afirman que una marca es un constructo multidimensional que implica la fusión de todo tipo de valores que coinciden con el desempeño de los consumidores y las necesidades psicosociales. Los beneficios que provocan son relativamente concretos $y$, por lo tanto, pueden comunicarse a los consumidores de manera sencilla y clara (Tybout y Calkins, 2005: 19). Sin embargo, puesto que el valor funcional puede ser fácilmente copiado por los competidores, los valores desempeñan un papel más importante en el proceso de branding, ya que tiene en cuenta las mentes, los corazones y el espíritu de los consumidores (Kotler, Kartajaya y Setiawan, 2010:52). Por lo tanto, el valor de la marca debe apelar a las necesidades emocionales de los consumidores e ir más allá de las funcionalidades y características del producto (Kotler, Bowen y Makens, 2010). En el caso concreto de los lugares, Kavaratzis y Ashworth (2005) consideran importante situarse en el punto de vista del usuario final en términos de la forma en que perciben, entienden, usan y conectan con el lugar.

La investigación reciente ha confirmado que los consumidores contemporáneos de hoy en día ya no están interesados en la simple compra de productos o servicios sino en adquirir una maravillosa experiencia emocional tal como indican Gobé (2001); O'Shaughnessy y O'Shaughnessy (2003); Brembeck y Ekstrom (2004); Morrison y Crane (2007). Esta experiencia emocional permite al consumidor forjar una profunda conexión permanente e íntima con la marca que trasciende la satisfacción material (Gobé, 2001: 27). Las experiencias aportan valores sensoriales, emocionales, cognitivos, conductistas y de relación que sustituyen a los valores funcionales (Schmitt, 2000). 
La marca como concepto fue visto como relevante, pero no siempre se ha entendido o se ha aplicado de manera efectiva (Kavaratzis, 2004: 65). En la sociedad actual, una marca sólida, tiene dos propósitos principales. Por un lado, como un mecanismo estratégico utilizado para captar la atención de los clientes potenciales y crear un vínculo emocional con ellos, diferenciando los productos/servicios con el fin de llegar a un determinado público objetivo. Por otro lado, como una marca o una identidad corporativa que protege los derechos de los propietarios para comercializar y vender la marca (Dias y Marques, 2011: 82). Por ello, el papel de la alta dirección se dirige a garantizar la visión estratégica, la cultura corporativa y la imagen de marca (Hankinson, 2007).

Esta estrategia de comunicación visual ha capturado la atención de las Organizaciones de Marketing de Destinos (DMO) buscando crear marcas de destino atractivas y atrayentes con el fin de alejarse de la multitud. Kotler y Gertner (2004) definen el branding de destinos como la creación, mantenimiento y potenciación de la marca de un lugar con el objetivo de generar una identidad propia frente al mercado internacional. Se utiliza el conjunto de actividades de marketing para apoyar la creación de un nombre, símbolo, logo, palabra, marca u otro gráfico para fácilmente identificar y diferenciar un destino transmitiendo, constantemente, las expectativas de una memorable experiencia de viaje que está especialmente asociada al destino, que sirve para consolidar y reforzar la conexión emocional entre el visitante y el territorio y, para reducir, los costes de búsqueda y riesgo percibido (Blain, Levy y Ritchie, 2005: 337). En un mercado cada vez más competitivo, las DMO deben generar valor a sus visitantes a través de marcas destino que dispongan de una personalidad atractiva (Hankinson, 2004).

Cuando el patrimonio cultural y los rasgos históricos se unen al proceso de branding, es más probable que se confíe en la marca. La manifestación de los antecedentes culturales del destino y la personalidad proporcionan una experiencia de viaje incomparable. Por lo tanto, un mayor uso de la cultura local y las tradiciones pueden servir como instrumentos de comunicación válidas para proyectar y presentar los valores fundamentales del destino y sus características únicas (Walker, 2010: 22). Al honrar a la comunidad de acogida con una identidad visual que refleja su identidad cultural, la historia y el patrimonio, se eleva la probabilidad de la marca de ser reconocidos en el mercado exterior (Evan, 2009). En este sentido, una marca con falta de autenticidad puede correr el riesgo de disolución con el tiempo, sobre todo, si la población local no se identifica con las características trasmitidas o siente una insuficiente representatividad de su identidad social (Wang, 1999).

La autenticidad, es por lo tanto un concepto crucial a considerar en la relación entre la marca y la identidad cultural e histórica, en la formación de la identidad visual (Walker, 2010: 24). En este sentido, se sugiere que un destino que quiere crear una identidad visual auténtica, 
debe rendir homenaje al concepto intangible de la identidad social, la integridad y la autenticidad que se han desarrollado históricamente en el tiempo (Gartner, 1996: 462).

\subsection{La estrategia de branding en Cabo Verde}

Desde que comenzó el auge del turismo en la isla de Sal en la década de 1960, Cabo Verde ha luchado por ganar una posición competitiva en el mercado internacional aunque, en 2009, aún no disponía de un plan de marketing turístico. Dada la percepción que las instituciones públicas están desarrollando de la relevancia del turismo para el crecimiento de las distintas comunidades geográficas, así como la toma de conciencia de la demanda social en torno a las actividades turísticas como factor de ocio, potencian el auge de la comunicación publicitaria en esta línea (Alonso González, 2007: 92).

Es, a partir de entonces, cuando el Ministerio de Turismo (MTIE) y la Dirección General de Turismo (DGT) elaboran el primer documento oficial para promocionar el país como destino turístico (Plan de Marketing 2011-2013). En el mismo, subyace la idea fundamental de transmitir la posibilidad de vivir experiencias en el archipiélago, ya sean culturales o de aventura. El plan de marketing se desarrolló principalmente para identificar los recursos existentes en el país, las potencialidades y limitaciones que se presentan, los atributos diferenciados, y definir una estrategia básica para posicionar estratégicamente la marca Cabo Verde como un competidor global (DGT, 2010b: 9). Al mismo tiempo, trabajan para crear una marca atractiva con la capacidad de conectarse emocionalmente con los consumidores actuales y potenciales, y comunicar su identidad cultural, sus acontecimientos históricos y sus patrimonios tangibles e intangibles. Establecen una estrategia de desarrollo de marcas para diferenciar los productos y servicios que ofrecen.

El desarrollo de la marca Cabo Verde comenzó con un análisis de las principales partes interesadas, es decir, representantes nacionales e internacionales de la industria turística, que proporcionan consejos y recomendaciones. La composición de este esfuerzo coordinado abarca también la participación de los ciudadanos locales. Con este fin, el proceso de branding prosigue con un concurso nacional para la creación y diseño de la marca turística. Entre el 25 de marzo y el 7 de mayo de 2010, 151 propuestas fueron validadas (DGT, 2010b: 41). El criterio que rigió la propuesta estableció las siguientes condiciones: el diseño del logotipo debe servir como una representación del pueblo de Cabo Verde; la diversidad de las diez islas; la tranquilidad y relajación (un destino seguro y rico en paisajes naturales); el sol que brilla durante todo el año; la belleza natural y playas exóticas; y la aproximación a Europa y a otros mercados emergentes.

Un jurado constituido por los representantes internacionales y miembros del MTIE, de la DGT y del IPDT (Instituto Portugués de Fomento del Turismo), eligieron una marca paraguas 
representativa con un logotipo colorista que refleja un país cosmopolita y moderno con capacidad para responder a las demandas futuras (figura 1).

\section{Análisis de la identidad y posicionamiento de la marca Cabo Verde}

Siguiendo a De Chernatony (2006), lo que diferencia a las marcas de un logotipo, es la presencia de un valor emocional y funcional. Estos valores emocionales y funcionales están intrínsecamente incorporados en los principios de la marca y representan los valores fundamentales de la organización (destino), así como responden a los deseos de los consumidores, con el fin de satisfacer sus necesidades. En el contexto del branding, como se ha indicado anteriormente, los valores funcionales y emocionales se pueden conectar con el patrimonio cultural y los valores históricos, como medio para incrementar la importancia de los valores intangibles, ofreciendo al cliente una experiencia inolvidable transmitida a través de una marca experiencial.

Como un intento de atraer a los potenciales visitantes ofreciendo una experiencia memorable, el MTIE emprendió la misión de crear la marca Cabo Verde con el objetivo de transformar la industria del turismo en un sector altamente competitivo mediante la creación de una identidad visual para representar al sector más rentable del país. Sin embargo, cabe destacar que, algunas partes interesadas, en especial la comunidad de acogida, son de la opinión de que la marca escogida no refleja la autenticidad del territorio ya que, el mismo enfoque se está utilizando en otros destinos (como se observa en la figura 2). La población siente que la marca Cabo Verde debe transmitir la experiencia, grandeza y riqueza de los valores del territorio: sus características, su diversidad, la personalidad de sus gentes, la energía, la magia, el carácter distintivo y la emoción. No se produce, por tanto, una identificación de los residentes con la imagen proyectada lo que se está convirtiendo tristemente en algo habitual en la planificación de destinos, como recientemente ha ocurrido entre los andaluces y Andalucía, en la campaña de 2008 (Guarinos, 2009: 45).

Por lo tanto, al ser una marca una potente herramienta de comunicación, debe mostrar las relaciones culturales, la riqueza y el carácter distintivo del lugar (Anholt, 2003). En este contexto, Hem y Iversen (2004) concluyen que las similitudes entre el logotipo y el destino son un factor determinante para mejorar el reconocimiento de la marca. El intento de crear una representación visual y una imagen atractiva del archipiélago a través del juego de colores y de las formas de las diez islas que lo componen, ensombreció el tratar de transmitir Ios elementos singulares que diferencian a Cabo Verde de la competencia creando una marca con identidad que generase fidelización y negocio.

3.1. Análisis de la identidad de la marca Cabo Verde 
Uno de los elementos principales de una marca es el logotipo porque transmite los valores y principios fundamentales recogidos en la estrategia de marketing y comunicación. A tal efecto, si una marca envía confusión a la hora de comunicar sus mensajes, el valor de marca generado para el consumidor, será desacertado. En el caso objeto de estudio, el diseño gráfico que se seleccionó provoca interpretaciones ambiguas lo que, a su vez, pone en duda la autenticidad de la marca. Por un lado, el enfoque es discriminatorio con los habitantes de las distintas islas pues, los colores son utilizados para representar a las personas que habitan estos territorios. Por lo tanto, la población de la isla de Santiago tiene un tono de piel más oscuro que la de San Vicente y esto es una falsa suposición. Por otro lado, el patrón secuencial de colores que se ha utilizado para la marca Cabo Verde es el mismo que el de otros destinos: Algarve, el Caribe y Andalucía (figura 2). Y, la mezcla y superposición tipográfica es una clara imitación de la marca de la aerolínea portuguesa TAP, lo que demuestra la falta de creatividad y autenticidad de la marca seleccionada para este destino.

La tabla 1 muestra las principales características que deben tenerse en cuenta durante la implementación de una estrategia de marca eficaz con el fin de crear un logotipo atractivo y atrayente que establezca vínculos emocionales con el cliente. Como se puede observar, la marca Cabo Verde carece de las características fundamentales de una marca diferenciada y única. Es una marca que carece de enfoque, de creatividad y autenticidad $y$, no se identifica con la comunidad de acogida, por lo que corre el riesgo de no ser aceptada por el mercado exterior. Además, tal como indican Olabarri y Quintana (2009: 3), resulta fundamental para el posicionamiento de un territorio, el no plantear acciones comunicativas y campañas puntuales sin considerar en cada momento de dónde se viene, dónde se está y cuáles habrán de ser las siguientes acciones a desarrollar.

\subsection{Análisis DAFO de la marca Cabo Verde}

El análisis DAFO es un instrumento ampliamente utilizado para diagnosticar situaciones, productos y servicios. Se opera identificando las debilidades y fortalezas, de carácter interno, y se confrontan con las amenazas y oportunidades que afectan a su desarrollo y cuyo origen resulta de aspectos relativos a la evolución del entorno, el cual condiciona de alguna forma la viabilidad de la actividad, mostrándose como tendencia a un futuro posible (Fernández, 2006). Se utiliza como herramienta estratégica en las etapas preliminares de la planificación (Bartol y Martin, 1991) pero también, para establecer acciones que propicien la incorporación de ventajas competitivas que aseguren la rentabilidad y el atractivo turísticos de un destino en casos de posicionamiento o reposicionamiento (Camisón y Monfort, 1998).

Los resultados (tabla 2) revelaron que Cabo Verde es un destino emergente con una elevada tendencia de crecimiento a nivel turístico consecuencia de la diversidad cultural y la oferta diversificada y, propiciado por la posición geográfica estratégica. Sin embargo, los limitados 
recursos financieros, la falta de mano de obra cualificada y la necesidad de un desarrollo de las infraestructuras sociales, pueden retrasar el desarrollo turístico del país. Entre sus fortalezas destacan la seguridad, la aproximación a mercados emergentes, el clima templado con sol y temperaturas suaves todo el año, y los paisajes únicos e inusuales.

Al ser un destino emergente con un alto índice de crecimiento del sector turístico en la última década, el Ministerio de Turismo debe tener en consideración las oportunidades, destacando: la liberalización del sector aéreo, el clima de inestabilidad política e inseguridad de los destinos competidores, y los nichos de mercado que demandan productos ofertados por Cabo Verde. Sin embargo, como cualquier otro destino que compite en el mercado mundial, hay obstáculos que se han identificado como amenazas, entre otros: la proximidad a los principales destinos emisores, la falta de reconocimiento de la identidad política, social, económica y laboral del turismo o la falta de alojamiento para responder a la demanda turística.

Con el fin de comprender plenamente las capacidades y potencialidades del sector turístico del país, Kotler y Gertner (2004: 46) sugieren, que este proceso requiere de la comprensión de las fuerzas ambientales que puedan afectar a la comercialización, en concreto, las fortalezas y debilidades del destino para competir con otros: el tamaño del mercado interno, el acceso a zonas comerciales regionales, el nivel educativo de la población, los incentivos fiscales, la mano de obra cualificada, el coste de los cambios laborales, de seguridad y demográficos.

\section{Conclusiones}

Este estudio ha proporcionado una visión del rápido crecimiento generado en el sector turístico de Cabo Verde. Debido a su posición geoestratégica que conecta África, Europa y las Américas, en el trascurso de tan sólo una década, el sector del turismo se ha erigido en una industria sostenible con capacidad de competir en el mercado internacional. Los datos de la Organización Mundial del Turismo para el año 2011 en el archipiélago muestran una contribución directa de viajes y turismo al PIB del país de 2,8 millones de euros lo que equivale al 16,2\% del PIB nacional. Además, ha generado para dicho año 28.500 empleos directos $(14,4 \%)$. Al reconocer esta importante contribución, el Gobierno de Cabo Verde ha dado prioridad a todas las actividades relacionadas con el turismo con el fin de aprovechar las oportunidades que el sector ofrece.

Debido a que los territorios tienen que ser capaces de valorar e impulsar sus propios activos de forma atractiva, conocer bien sus diferencias y explotarlas, el Ministerio de Turismo puso en marcha en 2010 el diseño de la marca y de la identidad visual para posicionar el destino en los circuitos internacionales. Sin duda, la creación de la marca Cabo Verde fue un logro 
importante en lo que respecta a la formulación de estrategias competitivas y de promoción. Sobre la base de un concurso nacional en el que se presentaron 151 propuestas se trató de involucrar a todos los actores principales. La marca actual de Cabo Verde fue seleccionada por un panel de jurados nacionales e internacionales elaborado por la Dirección General de Turismo para representar la identidad turística del país. Sin embargo, el diseño de la marca seleccionada, como se ha demostrado a través del análisis, no cumple con los criterios para constituirse como una marca auténtica pues no ha recibido la aprobación de la comunidad local al no respetar a los pobladores de las distintas islas que componen el archipiélago ya que genera discriminación entre ellos. Por lo tanto, no se ha tenido en cuenta a uno de los principales grupos de interés a la hora de crear una marca turística y de generar valor, los residentes como protagonistas de las estrategias de endomarketing. Además, se constata la falta de creatividad y de autenticidad de la marca por la semejanza con los logotipos de otros destinos o empresas turísticas tales como Algarve, Andalucía, Caribe o TAP.

El análisis DAFO presentado revela los puntos fuertes y débiles del sector turístico, así como los retos que se deben superar para aprovechar las oportunidades que se presentan. La identificación de los puntos fuertes del destino con el fin de eliminar las amenazas es un criterio fundamental para el desarrollo de una estrategia de marca eficaz. La comprensión de cómo aprovechar las oportunidades para reducir la debilidad es una tarea difícil que abarca, una colaboración proactiva y un enfoque integral de todos los actores y grupos de interés involucrados. Con este fin, todos estos factores deben tenerse en cuenta para plantear un necesario nuevo enfoque para desarrollar una identidad visual que resalte la autenticidad y diferenciación del destino Cabo Verde.

\section{REFERENCIAS BIBLIOGRÁFICAS}

AAKER, David Allen (1991): Managing Brand Equity: Capitalizing On The Value Of A Brand. New York: The Free Press.

ALONSO FERNÁNDEZ, Carmen María (2007): "La construcción de la imagen turística de un territorio a través de la publicidad", Questiones Publicitarias, Vol. I, no 12. pp. 91-104.

ANHOLT, Simon (2003): "Branding Places And Nations", In Clifton, Rita y Simmons, John (EDS), Brands and Branding. London: Profile Books LTD.

BARTOL, Kathryn M. y MARTIN, David C. (1991): Management. New York, McGraw Hill, Inc.

BLAIN, Carmen, LEVY, Stuart E. Y RITCHIE, J. R. Brent (2005): "Destination Branding: Insights And Practices From Destination Management Organizations", Journal of Travel Research, Vol. 43, no 4, pp. 328-338. 
BREMBECK, Helene Y EKSTROM, Karin (2004): Elusive Consumption. Oxford: Berg Publishers.

CAMISÓN, César y MONFORT, Vicente (1998): "Estrategias de reposicionamiento para destinos turísticos maduros: El caso de la Costa Blanca", Estudios Turísticos, no 135, pp. 5-28.

DE CHERNATONY, Leslie (2006): From Brand Revision To Brand Evaluation: The Strategic Process Of Growing And Strengthening Brands. 3rd Ed. Oxford, Elsevier.

DE CHERNATONY, Leslie y DOLL' OLMO RILIEY, Francesca (1998): "Experts' Views About Defining Service Brands And The Principles Of Services Branding", Journey of Business Research, Vol. 46, pp. 181-192.

DIAS, Francisco Y MARQUES, Isabel Andrés (2011): "One Flew Over The Brands' Nest: Rethinking Destination Brand", European Journal of Tourism, Hospitality and Recreation, Vol. 2 (3), pp. 79-104.

DINNIE, Keith (2008): Nation Branding: Concepts, Issues, Practice. Burlington Massachusetts: Butterworth-Heinemann.

DIRECCIÓN GENERAL DEL TURISMO (DGT) (2010a): Plano Estratégico para o Desenvolvimento de Turismo em Cabo Verde 2010-2013. Praia - Cabo Verde, Ministério de Economia, Crescimento e Competitividade.

DIRECCIÓN GENERAL DEL TURISMO (DGT) (2010b): Plano de Marketing para o Turismo de Cabo Verde 2011-2013. Praia - Cabo Verde, Ministério do Turismo, Industria e Energia e Instituto Portuguese de Turismo.

EVAN, Graeme (2009): "Creative Cites, Creative Spaces, And Urban Policy", Urban Studies, Vol. 46 (5 \& 6), pp. 1003-1040.

FERNÁNDEZ, Carlos (2006): "El Turismo Rural en el Desarrollo Local", pp. 575-612, En: HTTP://WWW.REDCANARIARURAL.ORG/PDF/26_FERNANDEZ_HERNANDEZ.PDF .

GARTNER, William C. (1996): Tourism Development: Principles, Processes, And Policies. New York: John Wiley \& Sons Inc.

GOBÉ, Marc (2001). Emotional Branding. New York: Allworth Press.

GUARINOS, Virginia (2009): "Questiones sobre la representación audiovisual de la nueva Andalucía. De la amistad al amor: Campaña Andalucía te quiere (2008)", Questiones Publicitarias, Vol. I, no 14. pp. 34-48.

HALL, C. Michael (2010): "Tourism Destination Branding And Its Effects On National Branding Strategies: Brand New Zealand, Clean And Green But Is It Smart?", European Journal of Tourism, Hospitality and Recreation, Vol. 1, no 1 , pp. 68-89. 
HANKINSON, Graham (2004): "The Brand Images Of Tourism Destinations: A Study Of The Saliency Of Organic Images", Journal of Product \& Brand Management, Vol. 13, no 1, pp. 6-14.

HANKINSON, Graham (2007): "The Management Of Destination Brands: Five Guiding Principles Based On Recent Developments In Corporate Branding Theory", Brand Management, Vol. 14, no 3, pp. 240-254.

HEM, Leif y IVERSEN, Nina (2004): "How To Develop A Destination Brand Logo: A Qualitative And Quantitative Approach", Scandinavian Journal of Hospitality and Tourism, Vol. 4, no 2, pp. 83-106.

KAVARATZIS, Mihalis (2004): "From City Marketing To City Branding: Towards A Theoretical Framework For Developing City Brands", Place Branding, Vol. 1, no 1 , pp. 58-73.

KAVARATZIS, Mihalis y ASHWORTH, G.J. (2005): "City Branding: An Effective Assertion Of Identity Or A Transitory Marketing Trick?", Tijdschrift Voor Economische en Sociale Geografie, Vol. 96, n 5, pp. 506-514.

KELLER, Kevin Lane (2003): Strategic Brand Management: Building, Measuring And Brand Equity, 2nd Ed. New Jersey: Upper Saddle River, Prentice Hall.

KOTLER, Philip y GERTNER, David (2004): "Country As Brand, Product And Beyond: A Place Marketing And Brand Management Perspective": In MORGAN, Nigel; PRITCHARD, Annette Y PRIDE, Roger, (Eds). Destination Branding: Creating The Unique Destination Proposition. Massachusetts: Elsevier ButterworthHeinemann.

KOTLER, Philip, BOWEN, John y MAKENS, James (2010): Marketing For Hospitality And Tourism. New Jersey: Pearson Prentice Hall.

KOTLER, Philip, KARTAJAYA, Hermawan y SETIAWAN, Iwan (2010): Marketing 3.0: From Product To Customer To The Human Spirit. New Jersey: John Wiley, Inc.

LYNCH, Joanne y De Chernatony, Leslie (2004): "The Power Of Emotion: Brand Communication In Business-to-Business Markets" Journal of Brand Management, Vol. 11 (5), pp. 403-419.

MITCHELL, Jonathan (2008): "Tourism Development In Cape Verde: The Policy Challenge Of Coping With Success". London: Overseas Development Institute.

MORRISON, Sharon y CRANE, Frederick G. (2007): "Building The Service Brand By Creating And Managing An Emotional Brand Experience", Brand Management, Vol. 14, no 5, pp. 410-421.

MUÑIZ, Norberto y CERVANTES, Miguel (2010): "Marketing de ciudades y Place Branding", Pecvnia, pp. 123-149. 
OLABARRI, Elena y QUINTANA, Miguel Ángel (2009): "Eficacia de las campañas publicitarias: El caso de las campañas de Turismo del Gobierno Vasco", Questiones Publicitarias, Vol. I, no 14. pp. 1-19.

ORGANIZACIÓN MUNDIAL DEL TURISMO (2013): "UNWTO World Tourism Barometer" Madrid, World Tourism Organization.

O'SHAUGHNESSY, John Y O'SHAUGHNESSY, Nicholas Jackson (2003): The Marketing Power Of Emotion. New York, Oxford University Press.

SCHMITT, Bernd (2000): Experiential Marketing. Barcelona, Deusto.

TKACHUK, Carolina (2008): "Marketing Turístico. Principios para el desarrollo estratégico de ciudades turísticas", En: http://hm.unq.edu.ar/archivos_hm/CT_MarketingTuristico(Rosario).pdf

TYBOUT, Alice M. y CALKINS, Tim (2005): Kellogg On Branding. Hoboken, New York: John Wiley \& Sons.

WALKER, Marian (2010): "Cities As Creative Spaces For Cultural Tourism: A Plea For The Consideration Of History": PASOS Revista de Turismo y Patrimonio Cultural, Vol. 8 (3) Special Issue, pp. 17-26.

WANG, Ning (1999): "Rethinking Authenticity In Tourism Experience", Annual of Tourism Research, Vol. 26, (2), pp. 349-370. 


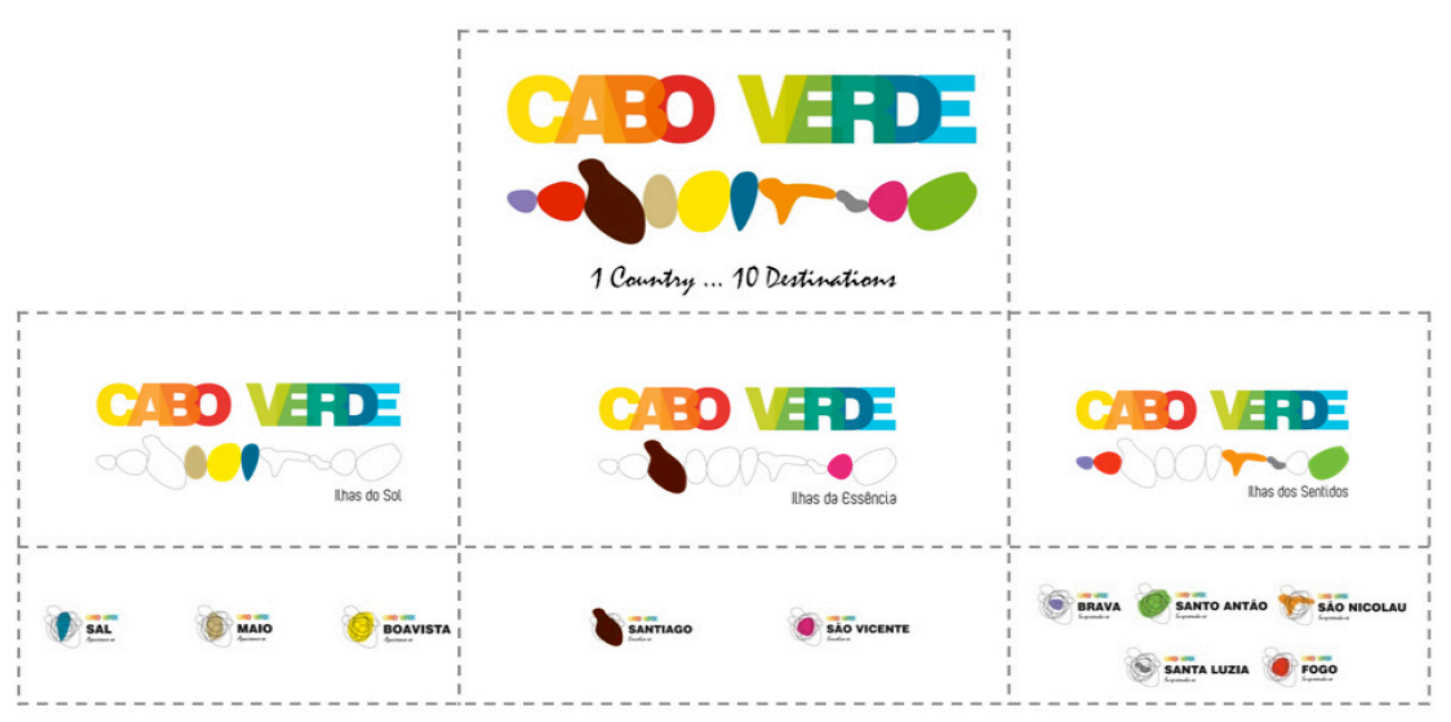

Figura 1: Marca Cabo Verde

Fuente: DGT (2010a)

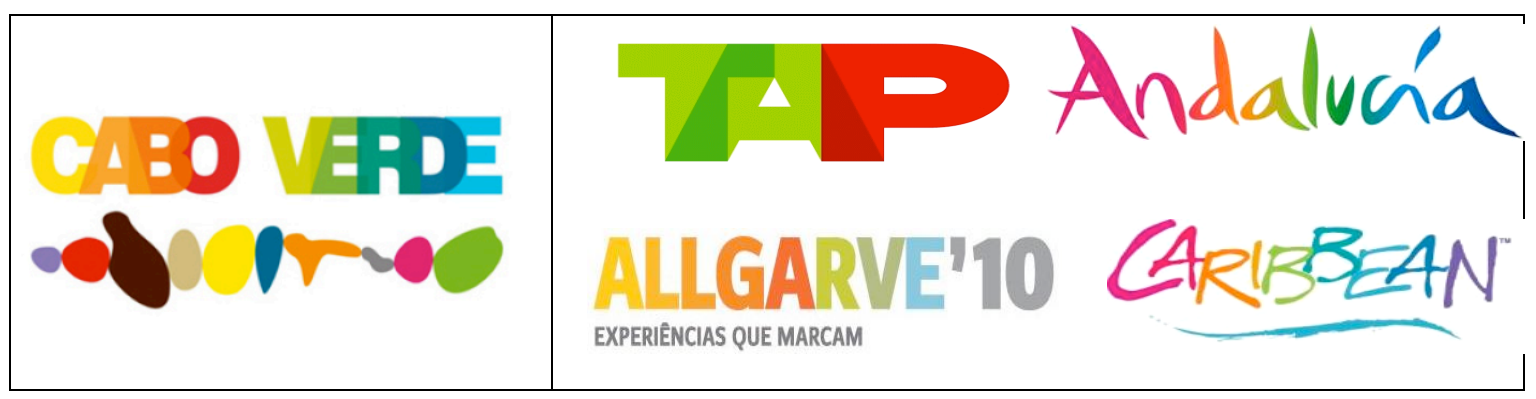

Figura 2: Las marcas que se asemejan a la marca Cabo Verde Fuente: DGT (2010b) y Google Imágenes 


\begin{tabular}{|c|c|c|}
\hline \begin{tabular}{ll}
\multicolumn{1}{c}{ Marca } \\
- & Autenticidad \\
Diferenciación de \\
productos y servicios \\
Recoger la historia de \\
la organización \\
- $\quad$ Poder registrarse \\
Poseer valores \\
funcionales y \\
emocionales \\
Herramienta de \\
comunicación \\
Tocar los corazones, \\
la mente y el espíritu \\
Forjar relaciones \\
duraderas \\
Trascender la \\
satisfacción material \\
Enfoque holístico
\end{tabular} & $\begin{array}{l}\text { Marcas de destino } \\
\text { - } \quad \text { Herramienta de } \\
\text { comunicación visual que } \\
\text { proyecto los valores } \\
\text { fundamentales y las } \\
\text { características únicas } \\
\text { - } \quad \text { Recoger la historia de un } \\
\text { destino } \\
\text { - Transmitir la experiencia } \\
\text { de un viaje memorable } \\
\text { Refuerzan la conexión } \\
\text { emocional } \\
\text { - } \text { Reducir el proceso de } \\
\text { búsqueda } \\
\text { - } \quad \text { Coducir el riesgo percibido } \\
\text { cultura, la historia y el } \\
\text { - } \quad \text { Manifrimonio } \\
\text { - } \quad \text { Hontecedenta la cultura local } \\
\text { Reflejan la comunidad de } \\
\text { acogida }\end{array}$ & $\begin{array}{ll}\text { Marca Cabo Verde } \\
\text { - } & \text { Falta autenticidad } \\
& \text { ho recoge la } \\
& \text { destoria del } \\
\text { - } & \text { Es similar a otras } \\
\text { - } & \text { Farcas } \\
\text { - } & \text { No transmite una } \\
& \text { experiencia } \\
\text { - } & \text { Falta enfororable } \\
\text { - } & \text { No se ha } \\
& \text { construido ni } \\
& \text { sobre la cultura } \\
\text { ni sobre la } \\
\text { historia } \\
\text { Es discriminatoria } \\
\text { No refleja la } \\
\text { comunidad de } \\
\text { acogida }\end{array}$ \\
\hline
\end{tabular}

Tabla 1: Desarrollo de las características de una marca

Fuente: Elaboración propia 


\section{FORTALEZAS}

- Plan de Marketing;

- Plan Estratégico de Desarrollo Turístico;

- Destino en expansión;

- Diversidad y herencia cultural;

- Destino emergente;

- Diversidad de oferta turística;

- Seguridad (destino seguro);

- Aproximación a mercados emergentes

- Clima templado;

- Paisaje único e inusual;

- Ubicación geográfica estratégica.

\section{DEBILIDADES}

- Objetivos inespecíficos en términos de marketing y promoción;

- Imagen de marca pobre;

- Inexistencia de estrategia de comunicación;

- Errónea imagen de marca;

- Problemas de autenticidad y creatividad;

- El Plan de Marketing no se corresponde con el Plan Estratégico para el Desarrollo del Turismo (PEDT);

- No hay una dirección estratégica definida;

- Recursos financieros limitados;

- Falta de mano de obra cualificada;

- Necesidad de desarrollo de infraestructuras sociales;

\section{OPORTUNIDADES}

- Fomentar la colaboración en inversion;

- Incentivos para empresarios;

- Crecimiento rápido del mercado;

- Liberalización gradual del transporte aéreo;

- Clima de inseguridad experimentada en destinos competidores;

- Inestabilidad política en destinos competidores;

- Aproximación a los principales mercados.

\section{AMENAZAS}

- No hay plan de comunicación para la marca actual:

- La marca actual no refleja a la comunidad de acogida;

- Falta estrategia de comunicación online;

- No se utiliza Internet como herramienta promocional;

- Excesiva dependencia del turismo internacional;

- Nuevas tasas impositivas al turismo;

- Proximidad a los principales destinos emisores;

- Falta de reconocimiento de la identidad política, social, económica y laboral;

- Falta de alojamiento

Tabla 2: Análisis DAFO de la marca Cabo Verde

Fuente: Elaboración propia

[Recibido: 10 de octubre de 2013. Aceptado con cambios: 2 de noviembre de 2014 .

Aceptación definitiva: 26 de noviembre de 2014]. 